\title{
Amplitude analyses and results on three-body charmless hadronic $B$ decays from $\mathrm{LHCb}$
}

\author{
Louis Henry* \\ Laboratoire de Physique Nucléaire et des Hautes Energies (LPNHE), Paris \\ E-mail: louis.henry@cern.ch
}

Charmless $b$-hadron decays in the Standard Model are characterised by tree amplitudes that are in general comparable in size to loop amplitudes, and potentially by New Physics amplitudes. $C P$-violation measurements using Dalitz-plot analyses in multi-body decays enable the various contributions to be disentangled. We present the most recent measurements in this sector, notably results on $B_{c}^{+} \rightarrow K^{+} K^{-} \pi^{+}, B_{d, s} \rightarrow \phi \pi^{+} \pi^{-}$, and suppressed $B^{0} \rightarrow 3 h$ decays, where $h$ is a kaon or a pion.

38th International Conference on High Energy Physics

3-10 August 2016

Chicago, USA

${ }^{*}$ Speaker.

$\dagger$ On behalf of the LHCb collaboration. 


\section{Introduction}

The study of charmless $b$-hadron decays allows to put constraints on New Physics (NP) by studying loop-dominated or annihilation-dominated transitions. Indeed, the suppression of tree-level $b \rightarrow u$ amplitudes allows for these transitions to compete with or even dominate tree diagrams.

The study of hadronic three-body charmless transitions allows to access to a wealth of physics observables. Indeed, these decays can originate from several quasi-two-body (Q2B) decays that interfere, giving information on the relative phases between these contributions. Additionally, $C P$ violation effects can arise from the interference between different Q2B contributions. Several techniques can be used in the analysis of three-body decays, such as the Dalitz plot in the case of a fully pseudo-scalar final state, or angular analyses in other cases.

The LHCb detector ([1] ) is located at the LHC accelerator at the Centre Européen pour la Recherche Nucléaire (CERN) in Geneva. It is a single-arm forward spectrometer covering the pseudorapidity range $2<\eta<5$, designed for the study of particles containing $b$ or $c$ quarks.

Among other features of the LHCb detector, analyses of charmless hadronic three-body decays benefit from the excellent tracking resolution of, typically, $\delta_{p} / p \approx 0.5-1 \%$ ([纪), and on a $95 \%$ $K^{ \pm} / \pi^{ \pm}$separation over the $3-100 \mathrm{GeV} / c$ kinematical range. The results presented in this document are from analyses of the full $3 \mathrm{fb}^{-1}$ Run I dataset, collected at centre-of-mass energies of 7 and $8 \mathrm{TeV}$.

\section{Search for $B_{c}^{+} \rightarrow K^{+} K^{-} \pi^{+}$}

In the Standard Model, decays of $B_{c}^{+}$mesons with no $b$ or $c$ in the final and intermediary states can proceed only via $\bar{b} c \rightarrow W^{+} \rightarrow u \bar{q}(q=d, s)$. Calculations predict branching fractions in the range $10^{-8}-10^{-6}$ ([3, 目). Any significant improvement could indicate the presence of $\bar{b} c$ annihilations involving particles beyond the SM, such as a mediating charged Higgs boson ([5, 6]).

Searches for $B_{c}^{+}$meson decays to the $K^{+} K^{-} \pi^{+}$final state have been performed in the fiducial region $p_{T}(B)<20 \mathrm{GeV} / c$ and $2.0<y(B)<4.5$ ([7]). Evidence for the decay $B_{c}^{+} \rightarrow \chi_{c 0} \pi^{+}$is found with a significance of $4.0 \sigma$. This result can be compared to the measurement involving another charmonium mode, $\frac{\sigma\left(B_{c}^{+}\right)}{\sigma\left(B^{+}\right)} \times \mathscr{B}\left(B_{c}^{+} \rightarrow J / \psi \pi^{+}\right)=(7.0 \pm 0.3) \times 10^{-6}$ ([8, 9]). Figure 1$]$ shows the distribution of data events on the Dalitz plot. The $\chi_{c 0}$ region can be seen between the two red lines, while the annihilation region is located below the blue line.

An indication of $\bar{b} c$ weak annihilation with a significance of $2.4 \sigma$ is reported in the region $\mathrm{m}\left(K^{+} K^{-}\right)<1.834 \mathrm{GeV} / c^{2}$. We obtain the annihilation-only branching fraction $R_{\mathrm{an}, K K \pi}=$ $\left(8.0_{-3.8}^{+4.4}\right.$ (stat.) \pm 0.6 (syst.) $) \times 10^{-8}$. Additionally, a 90\%(95\%) confidence level (CL) upper limit $R_{\text {an }, K K \pi}<15(17) \times 10^{-8}$ is estimated by comparing profile likelihood ratios for the "signal+background" against "background-only" hypotheses.

\section{Search for $B_{d, s} \rightarrow \phi \pi^{+} \pi^{-}$}

The decays $B_{d, s} \rightarrow \phi \pi^{+} \pi^{-}$have not been observed before. They are examples of Flavour Changing Neutral Current (FCNC) decays, which provide a sensitive probe for the effect of physics beyond 

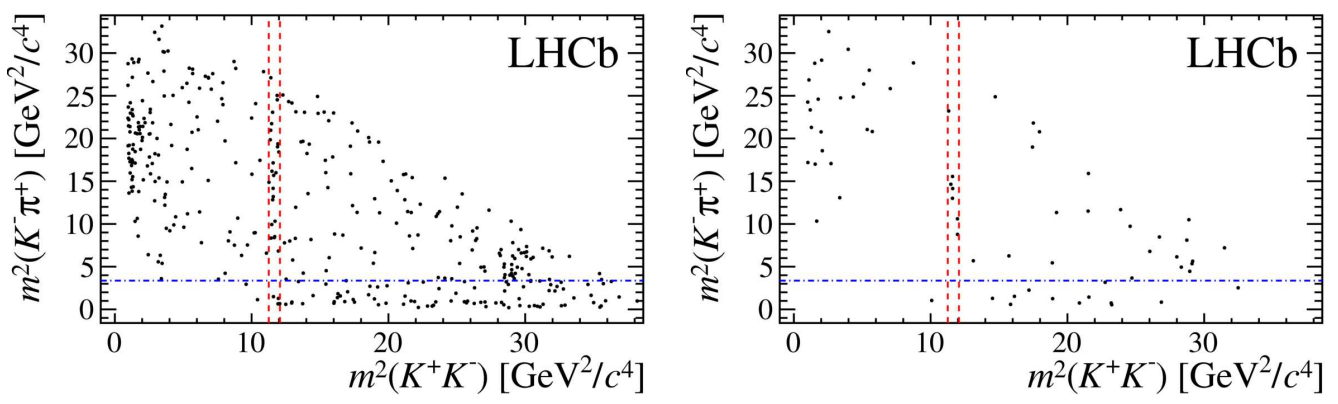

Figure 1: Distribution of events in the signal region $6.2<\mathrm{m}\left(K^{+} K^{-} \pi^{+}\right)<6.35 \mathrm{GeV} / c^{2}$ on the $m^{2}\left(K^{-} \pi^{+}\right)$ vs. $m^{2}\left(K^{+} K^{-}\right)$plane for (left) $\mathscr{O}_{\mathrm{BDT}}>0.12$ and (right) $\mathscr{O}_{\mathrm{BDT}}>0.18$, where $\mathscr{O}_{\mathrm{BDT}}$ is the output variable of a Boosted Decision Tree (BDT). The vertical red dashed lines represent a band of width $\pm 60 \mathrm{MeV} / c^{2}$ around the $\chi_{c 0}$ mass. The horizontal blue dot-dashed line indicates the upper bound of the annihilation region at $m\left(K^{-} \pi^{+}\right)=1.834 \mathrm{GeV} / c^{2}$, representing $17 \%$ of the available phase-space area.

the Standard Model because their amplitudes are described by loop (or penguin) diagrams where new particles may enter ([10]).

We report the first observation of the inclusive decay $B_{s}^{0} \rightarrow \phi \pi^{+} \pi^{-}$([1] $)$. The branching fraction in the invariant-mass range $400<\mathrm{m}\left(\pi^{+} \pi^{-}\right)<1600 \mathrm{MeV} / c^{2}$ is measured to be

$$
\mathscr{B}\left(B_{s}^{0} \rightarrow \phi \pi^{+} \pi^{-}\right)=(3.48 \pm 0.23 \pm 0.17 \pm 0.35) \times 10^{-6},
$$

where the uncertainties are statistical, systematic, and related to the $B_{s}^{0} \rightarrow \phi \phi$ control channel, respectively.

Evidence is also seen for the inclusive decay $B^{0} \rightarrow \phi \pi^{+} \pi^{-}$with a significance of $4.5 \sigma$. The branching fraction in the invariant-mass range $400<\mathrm{m}\left(\pi^{+} \pi^{-}\right)<1600 \mathrm{MeV} / c^{2}$ is measured to be

$$
\mathscr{B}\left(B^{0} \rightarrow \phi \pi^{+} \pi^{-}\right)=(1.82 \pm 0.25 \pm 0.41 \pm 0.14) \times 10^{-7} .
$$

An amplitude analysis is used to separate out exclusive contributions to the $B_{s}^{0}$ decays. Figure 2 shows the fit to background-subtracted distributions of angular variables in data events.

The decay $B_{s}^{0} \rightarrow \phi f_{0}(980)$ is observed with a significance of $8 \sigma$, and the branching fraction is

$$
\mathscr{B}\left(B_{s}^{0} \rightarrow \phi f_{0}(980), f_{0}(980) \rightarrow \pi^{+} \pi^{-}\right)=\left(1.12 \pm 0.16_{-0.08}^{+0.09} \pm 0.11\right) \times 10^{-6} .
$$

The decay $B_{s}^{0} \rightarrow \phi f_{2}(1270)$ is observed with a significance of $5 \sigma$, and the branching fraction is

$$
\mathscr{B}\left(B_{s}^{0} \rightarrow \phi f_{2}(1270), f_{2}(1270) \rightarrow \pi^{+} \pi^{-}\right)=\left(0.61 \pm 0.13_{-0.05}^{+0.12} \pm 0.06\right) \times 10^{-6} .
$$

There is also a contribution from higher mass $S$-wave $\pi^{+} \pi^{-}$states in the region $1350-1600 \mathrm{MeV} / c^{2}$, which could be described by a superposition of the $f_{0}(1370)$ and the $f_{0}(1500)$ resonances. There is $4 \sigma$ evidence for the decay $B_{s}^{0} \rightarrow \phi \rho^{0}$ with a branching fraction of

$$
\mathscr{B}\left(B_{s}^{0} \rightarrow \phi \rho^{0}, \rho^{0} \rightarrow \pi^{+} \pi^{-}\right)=(2.7 \pm 0.7 \pm 0.2 \pm 0.2) \times 10^{-7} .
$$

This is lower than the Standard Model prediction of $\left(4.4_{-0.7}^{+2.2}\right) \times 10^{-7}$ ([12]), but still consistent with it, and provides a constraint on possible contributions from NP in this decay. 

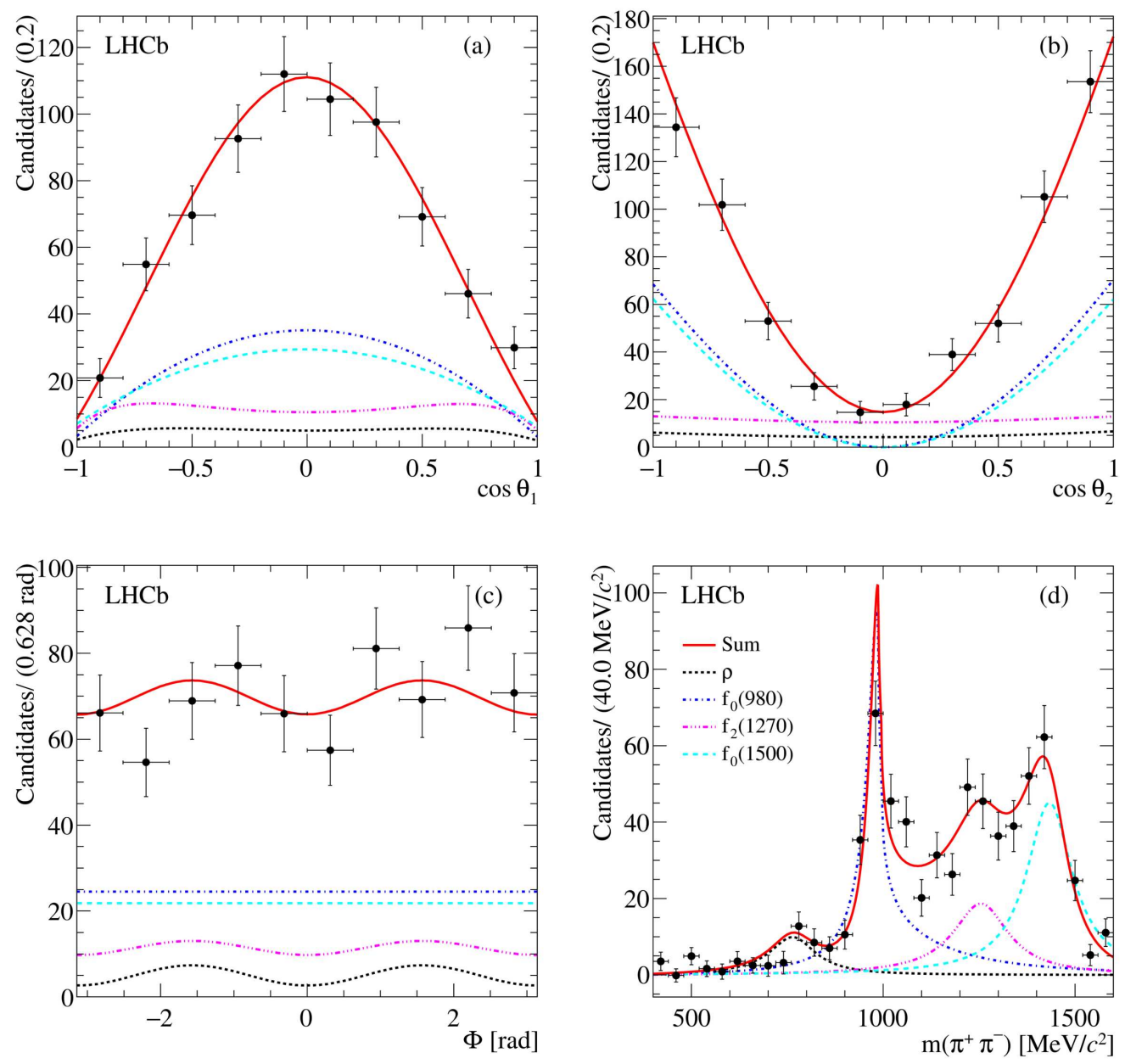

Figure 2: Projections of (a) $\cos \left(\theta_{1}\right)$, (b) $\cos \left(\theta_{2}\right)$, (c) $\Phi$, and (d) $m\left(\pi^{+} \pi^{-}\right)$for the best fit to backgroundsubtracted $B_{s}^{0} \rightarrow \phi \pi^{+} \pi^{-}$events. The $\rho^{0}$ contribution is shown by the dotted black line, the $f_{0}(980)$ by the dot-dashed blue line, the $f_{2}(1270)$ by the double-dot-dashed magenta line and the $f_{0}(1500)$ by the dashed cyan line.

\section{Branching fractions of $B^{+} \rightarrow K^{+} K^{+} \pi^{-}$and $B^{+} \rightarrow \pi^{+} \pi^{+} K^{-}$}

Transitions of the type $b \rightarrow s s \bar{d}$ and $b \rightarrow d d \bar{s}$ are rare in the SM ([13, 14]). The calculation of the $b \rightarrow s s \bar{d}$ amplitude results in branching fractions of at most $\mathscr{O}\left(10^{-11}\right)$.

Including all statistical and systematic uncertainties, the ratios of branching fractions are calculated to be

$$
\begin{aligned}
& \frac{\mathscr{B}\left(B^{+} \rightarrow K^{+} K^{+} \pi^{-}\right)}{\mathscr{B}\left(B^{+} \rightarrow K^{+} K^{-} \pi^{+}\right)}=(-7.5 \pm 4.9 \pm 1.0) \times 10^{-3}, \\
& \frac{\mathscr{B}\left(B^{+} \rightarrow \pi^{+} \pi^{+} K^{-}\right)}{\mathscr{B}\left(B^{+} \rightarrow \pi^{+} \pi^{-} K^{+}\right)}=(1.1 \pm 4.0 \pm 0.1) \times 10^{-4},
\end{aligned}
$$

where the uncertainties are statistical and systematic, respectively ([15]). To obtain upper limits on the branching fractions, the frequentist approach of Feldman and Cousins ([16]) is used to deter- 
mine $90 \%$ and $95 \%$ confidence region bands that relate the true values of the branching fractions to the measured numbers of signal events. These bands are constructed using the results of simulation studies that account for relevant biases in the fit procedure and include statistical and systematic uncertainties. The confidence region bands are shown in Fig. 3. The 90\% (95\%) confidence level
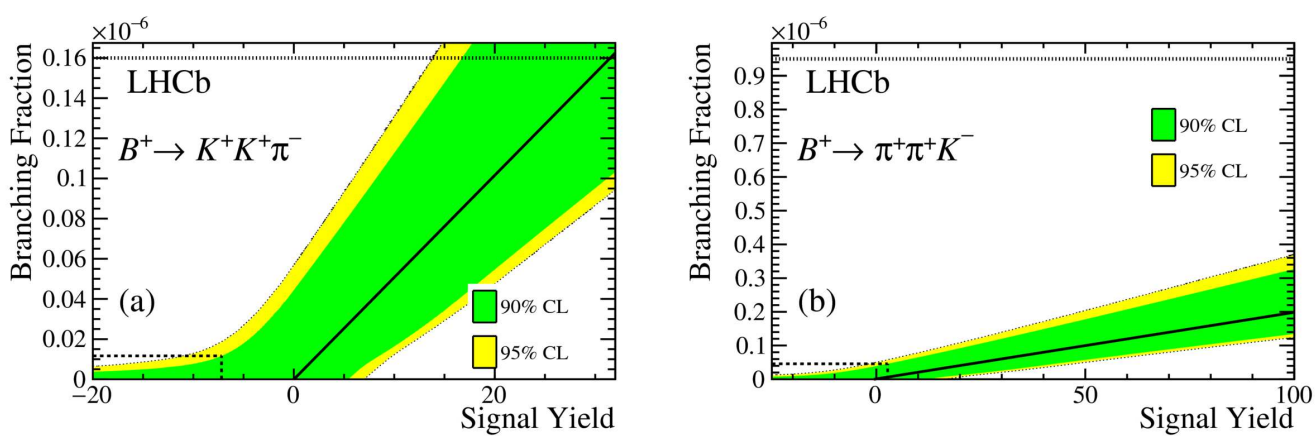

Figure 3: Feldman-Cousins 90\% (green) and 95\% (yellow) CL bands for (a) $B^{+} \rightarrow K^{+} K^{+} \pi^{-}$and (b) $B^{+} \rightarrow \pi^{+} \pi^{+} K^{-}$decays, including statistical and systematic uncertainties.

(CL) upper limits are found to be

$$
\begin{aligned}
& \mathscr{B}\left(B^{+} \rightarrow K^{+} K^{+} \pi^{-}\right)<1.1 \times 10^{-8}\left(1.8 \times 10^{-8}\right) \text { at } 90 \%(95 \%) \mathrm{CL}, \\
& \mathscr{B}\left(B^{+} \rightarrow \pi^{+} \pi^{+} K^{-}\right)<4.6 \times 10^{-8}\left(5.7 \times 10^{-8}\right) \text { at } 90 \%(95 \%) \mathrm{CL} .
\end{aligned}
$$

In summary, no evidence is found for the highly-suppressed decays $B^{+} \rightarrow K^{+} K^{+} \pi^{-}$and $B^{+} \rightarrow$ $\pi^{+} \pi^{+} K^{-}$, and upper limits are placed on their branching fractions. The results are approximately fourteen and twenty times more stringent than previous measurements ([17, 18, 19]) and constrain various extensions of the SM ([20, 21]).

\section{Conclusion}

The analysis of three-body hadronic charmless decays in LHCb already allows to set constraints on NP and to observe new channels of interest, such as $B_{d, s} \rightarrow \phi \pi^{+} \pi^{-}$, using the Run I data of LHCb. During the next few years, the increased datasets will allow to access to more observables, such as $\beta_{(s) \text {,eff }}$ and $\gamma$, using amplitude analyses.

\section{References}

[1] Jr. Alves, A. Augusto et al. The LHCb detector at the LHC. JINST, 3:S08005, 2008.

[2] Roel Aaij et al. LHCb Detector Performance. Int. J. Mod. Phys., A30(07):1530022, 2015.

[3] S. Descotes-Genon, J. He, E. Kou, and P. Robbe. Nonleptonic charmless $B_{c}$ decays and their search at lhcb. Phys. Rev. D, 80:114031, Dec 2009.

[4] Xin Liu, Zhen-Jun Xiao, and Cai-Dian Lü. Pure annihilation type $B_{c} \rightarrow M_{2} M_{3}$ decays in the perturbative qed approach. Phys. Rev. D, 81:014022, Jan 2010. 
[5] Wei-Shu Hou. Enhanced charged higgs boson effects in $B^{-} \rightarrow \tau \bar{v}, \mu \bar{v}$ and $b \rightarrow \tau \bar{v}+x$. Phys. Rev. $D$, 48:2342-2344, Sep 1993.

[6] Shinya Kanemura, Mariko Kikuchi, and Kei Yagyu. Fingerprinting the extended Higgs sector using one-loop corrected Higgs boson couplings and future precision measurements. Nucl. Phys., B896:80-137, 2015.

[7] Roel Aaij et al. Study of $B_{c}^{+}$decays to the $K^{+} K^{-} \pi^{+}$final state and evidence for the decay $B_{c}^{+} \rightarrow \chi_{c 0} \pi^{+} .2016$.

[8] Roel Aaij et al. Measurement of $B_{c}^{+}$production in proton-proton collisions at $\sqrt{s}=8 \mathrm{TeV}$. Phys. Rev. Lett., 114:132001, 2015.

[9] K. A. Olive et al. Review of Particle Physics. Chin. Phys., C38:090001, 2014.

[10] Martti Raidal. Cp asymmetry in $b \rightarrow \phi K_{S}$ decays in left-right models and its implications for $B_{S}$ decays. Phys. Rev. Lett., 89:231803, Nov 2002.

[11] Roel Aaij et al. Observation of the decay $B_{s}^{0} \rightarrow \phi \pi^{+} \pi^{-}$and evidence for $B^{0} \rightarrow \phi \pi^{+} \pi^{-} .2016$.

[12] Lars Hofer, Dominik Scherer, and Leonardo Vernazza. $B_{s} \rightarrow \phi \rho^{0}$ and $B_{s} \rightarrow \phi \pi^{0}$ as a handle on isospin-violating New physics. JHEP, 02:080, 2011.

[13] Dan Pirjol and Jure Zupan. Predictions for $b \rightarrow s s \bar{d}$, and $b \rightarrow d d \bar{s}$ decays in the SM and with new physics. JHEP, 02:028, 2010.

[14] Katri Huitu, Cai-Dian Lü, Paul Singer, and Da-Xin Zhang. Searching for new physics in $b \rightarrow s s \bar{d}$ decays. Phys. Rev. Lett., 81:4313-4316, Nov 1998.

[15] Roel Aaij et al. Search for the suppressed decays $B^{+} \rightarrow K^{+} K^{+} \pi^{-}$and $B^{+} \rightarrow \pi^{+} \pi^{+} K^{-} .2016$.

[16] Gary J. Feldman and Robert D. Cousins. A Unified approach to the classical statistical analysis of small signals. Phys. Rev., D57:3873-3889, 1998.

[17] Kazuo Abe et al. Study of three-body charmless B decays. Phys. Rev., D65:092005, 2002.

[18] Bernard Aubert et al. Measurements of the branching fractions and charge asymmetries of charmless three-body charged B decays. Phys. Rev. Lett., 91:051801, 2003.

[19] Bernard Aubert et al. Search for the highly suppressed decays $B^{-} \rightarrow K^{+} \pi^{-} \pi^{-}$and $B^{-} \rightarrow K^{-} K^{-} \pi^{+}$. Phys. Rev., D78:091102, 2008.

[20] S. Fajfer and P. Singer. Search for new physics in Delta $\mathrm{S}=2$ two-body (VV, PP, VP) decays of the Bmeson. Phys. Rev., D62:117702, 2000.

[21] Svjetlana Fajfer, Jernej F. Kamenik, and Nejc Kosnik. $b \rightarrow d d \bar{s}$ transition and constraints on new physics in B-decays. Phys. Rev., D74:034027, 2006. 